\title{
A time-dependent jet model for the emission from Sagittarius $A^{*}$
}

\author{
D. Maitra ${ }^{1,2}$, S. Markoff ${ }^{1}$, and H. Falcke ${ }^{3,4}$ \\ 1 Astronomical Institute "Anton Pannekoek", University of Amsterdam, Science Park 904, 1098 XH Amsterdam, The Netherlands \\ 2 Department of Astronomy, University of Michigan, Ann Arbor, MI, 48109, USA \\ e-mail: dmaitra@umich.edu \\ 3 Institute for Mathematics, Astrophysics and Particle Physics, Radboud University, 6500 GL Nijmegen, The Netherlands \\ 4 ASTRON, Oude Hoogeveensedijk 4, 7991 PD Dwingeloo, The Netherlands
}

Received 21 August 2009 / Accepted 3 November 2009

\begin{abstract}
Context. The source of emission from Sgr A*, the supermassive black hole at the Galactic Center, is still unknown. Flares and data from multiwavelength campaigns provide important clues about the nature of Sgr A* itself.

Aims. Here we attempt to constrain the physical origin of the broadband emission and the radio flares from Sgr A*.

Methods. We developed a time-dependent jet model, which for the first time allows one to compare the model predictions with flare data from Sgr A*. Taking into account relevant cooling mechanisms, we calculate the frequency-dependent time lags and photosphere size expected in the jet model. The predicted lags and sizes are then compared with recent observations.

Results. Both the observed time lags and size-frequency relationships are reproduced well by the model. The combined timing and structural information strongly constrain the speed of the outflow to be mildly relativistic, and the radio flares are likely to be caused by a transient increase in the matter channelled into the jets. The model also predicts light curves and structural information at other wavelengths which could be tested by observations in the near future.

Conclusions. We show that a time-dependent relativistic jet model can successfully reproduce: (1) the quiescent broadband spectral energy distribution of Sgr A*; (2) the observed 22 and $43 \mathrm{GHz}$ light curve morphologies and time lags; and (3) the frequency-size relationship. The results suggest that the observed emission at radio frequencies from Sgr $\mathrm{A}^{*}$ is most easily explained by a stratified, optically thick, mildly relativistic jet outflow. Frequency-dependent measurements of time-lags and intrinsic source size provide strong constraints on the bulk motion of the jet plasma.
\end{abstract}

Key words. black hole physics - Galaxy: center - galaxies: active - galaxies: jets - radiation mechanisms: general radio continuum: general

\section{Introduction}

The compact radio source Sagittarius A* (hereafter Sgr A*), at an estimated distance of $8.4 \pm 0.6 \mathrm{kpc}$ (Reid et al. 2009) and mass of $\sim 4 \times 10^{6} M_{\odot}$ (Ghez et al. 2008; Gillessen et al. 2009), is our closest supermassive black hole. When compared to other active galactic nuclei (AGN), Sgr A* is extremely under-luminous at all wavelengths, suggesting either that the mass accretion rate $(\dot{M})$ onto the black hole, or the radiative efficiency, is very low. Polarization measurements suggest that $\dot{M} \leq 10^{-7} M_{\odot} /$ yr (Marrone et al. 2007). Recent multiwavelength campaigns that have monitored Sgr A* simultaneously from radio to $\mathrm{X}$-rays have found that even though the luminosity of Sgr A* is low relative to its AGN counterparts, its emission is quite variable at all wavelengths. Flaring activity at both sub-mm and higher frequencies has been observed on timescales ranging from minutes to hours, suggesting that the emission at these frequencies is produced very close to the supermassive black hole, probably within a few tens of gravitational radii $\left(R_{\mathrm{g}}=G M / c^{2}\right)$. Although flaring appears to be nearly simultaneous at these frequencies (Marrone et al. 2008; Eckart et al. 2008; Dodds-Eden et al. 2009), the flares seem to have an increasingly long timedelay at longer wavelengths. For example, Yusef-Zadeh et al. (2008) found that the $43 \mathrm{GHz}$ flares precede the $22 \mathrm{GHz}$ flares by $20 \pm 10 \mathrm{~min}$, and that $350 \mathrm{GHz}$ flares precede those of $43 \mathrm{GHz}$ by $30-60 \mathrm{~min}$.
The physical origin of the observed electromagnetic radiation from $\mathrm{Sgr} \mathrm{A}^{*}$ has been under active debate for more than a decade. Attempts to model the emission from Sgr A* can be broadly classified into two categories: (1) accretion inflow models (Melia 1992; Narayan et al. 1998; Liu \& Melia 2002; Yuan et al. 2006); and (2) relativistic outflow/jet models (Falcke \& Biermann 1999; Falcke \& Markoff 2000; Yuan et al. 2002). The radio, NIR, and X-ray flare light curves were modeled assuming expansion of a spherical plasma blob (see e.g., Yusef-Zadeh et al. 2008; Eckart et al. 2009). Recently Falcke et al. (2009) suggested that the radio time-lag data and measurements of intrinsic size at various frequencies (e.g., Bower et al. 2004; Shen et al. 2005; Doeleman et al. 2008) can be used to resolve the degeneracy between inflow and outflow models. It has long been suspected that radio flares such as those reported by Yusef-Zadeh et al. (2008) could be "smoking gun evidence" of jets in sources such as Sgr A* (Falcke 1999b).

While jets have not been directly imaged in Sgr A*, several observations strongly suggest the presence of jets in this source. The flat/slightly inverted radio spectral energy distribution (SED) (signature of a compact self-absorbed jet in the radio; see e.g., Blandford \& Königl 1979) observed from Sgr A* are similar to those of other low-luminosity AGNs. M 81 and Sgr A* have very similar spectra and polarization properties in the cm-radio band, and M 81 has been found to have weak jets (Nagar et al. 2005). Radio observations of the stellar X-ray 
binary system A0620-00 (Gallo et al. 2006) at almost near quiescent luminosity suggest that compact jets are present at bolometric luminosities as low as $\sim 10^{-7} L_{\text {Edd. }}$. If jet physics and accretion flow scale with the mass of the black hole, then Sgr A* $\left(L_{\text {bol }} \sim 10^{-9} L_{\text {Edd }}\right)$ is also expected to harbor a faint, compact jet. Steady-state jet models have previously been used to model the broadband quiescent spectra of Sgr A* successfully (Falcke \& Markoff 2000; Yuan et al. 2002); steady-state models, however, cannot predict the flaring activity.

In this paper we develop a proper treatment of the timedependent cooling processes of the particle distribution, while keeping the basic hydrodynamic outflow model the same as in Falcke (1996), allowing a semi-analytical treatment of the problem. Once dominant cooling mechanisms are taken into account, the time-dependent jet model naturally accounts for all the observational constraints, namely, (a) the broadband SED; (b) the observed light-curve morphologies and time-lags; and (c) the observed frequency-size relationship.

\section{Model}

We assume that a fraction of the accretion inflow is chanelled into two symmetric, collimated supersonic outflows. The energy distribution of the radiating leptons entering the jet is assumed to be a thermal, relativistic, Maxwell-Juttner distribution. Most of the kinetic energy of the jet is assumed to be carried by cold, non-radiating baryons. The bulk speed at the base of the jet is assumed to be the sound speed, and beyond the base (or the "nozzle") the jet plasma accelerates longitudinally via pressure gradient and expands laterally with the sound speed $\beta_{\mathrm{s}} \sim 0.4$. We adopt the standard one-fluid approximation used in magnetohydrodynamics (MHD; see e.g., Leismann et al. 2005a,b), and treat the magnetized jet plasma as a single-component fluid of adiabatic index $4 / 3$. The longitudinal velocity profile is obtained by solving the relativistic Euler equation, and the magnetic field strength is estimated from flux conservation (see Falcke 1996; Falcke \& Markoff 2000, for details of the hydrodynamics of a pressure-driven supersonic jet).

The observed spectrum from the compact jet is modeled as the sum of emission from cylindrical segments along the jet axis. Within each segment, we consider the following processes influencing the local evolution of the particle distribution:

- synchrotron cooling: Losses due to synchrotron emission are given by $\dot{\gamma}_{\mathrm{Syn}}=4 c \sigma_{\mathrm{T}} U_{B} \gamma^{2} /\left(3 m_{\mathrm{e}} c^{2}\right)$, where $\sigma_{\mathrm{T}}$ is the Thomson cross-section and $U_{B}=B^{2} / 8 \pi$ is the magnetic energy density;

- inverse Compton (IC) cooling: Losses due to IC scatterings are given by $\dot{\gamma}_{\text {Com }}=4 c\left\langle\sigma_{\mathrm{KN}} U_{\text {rad }}(t)\right\rangle_{\gamma} \gamma^{2} /\left(3 m_{\mathrm{e}} c^{2}\right)$, where, following de Kool et al. (1989), we define $\left\langle\sigma_{\mathrm{KN}} U_{\mathrm{rad}}(t)\right\rangle_{\gamma}=$ $4 \pi / c \int \sigma_{\mathrm{KN}}(\gamma, v) J_{v} \mathrm{~d} v$. Here $\sigma_{\mathrm{KN}}(v, \gamma)$ is the Klein-Nishina correction to the scattering cross-section and $J_{v}$ is the mean intensity;

- adiabatic expansion: Cooling due to adiabatic expansion is given by $\dot{\gamma}_{\text {ad }}=(1 / 3) \gamma \nabla \cdot v$, where $\nabla \cdot v$ denotes the divergence of the bulk velocity field (Longair 1992).

For numerical discretization, we assume that the plasma in each segment cools during the time that it requires to cross the segment. We follow the evolution of this "parcel" of jet plasma as it moves from one segment to another, i.e., in a comoving frame, without any particle loss/escape. For simplicity, we assume that this comoving "parcel" of particles cools and radiates independently, i.e., is not influenced by its neighbors. While this is obviously a simplification, given the overall simplicity of the model we feel this is justified. The continuity equation for the time evolution of leptons in this case is given by

$$
\frac{\partial N(\gamma, t)}{\partial t}=\frac{\partial}{\partial \gamma}[\dot{\gamma}(\gamma, t) N(\gamma, t)] ; \dot{\gamma}(\gamma, t)=\dot{\gamma}_{\mathrm{Syn}}+\dot{\gamma}_{\mathrm{Com}}+\dot{\gamma}_{\mathrm{ad}}
$$

Given the low luminosity of Sgr A* $\left(\sim 10^{-9} L_{\text {Edd }}\right)$, the compactness parameter $\left(\ell \equiv L \sigma_{T} /\left(\mathrm{Rm}_{\mathrm{e}} \mathrm{c}^{3}\right)\right.$; see Guilbert et al. 1983) is very small $\left(\ell \sim 10^{-6}\right.$ for $R=10 R_{\mathrm{g}}$ ). Therefore, pair processes are not important here. There is also no injection term in Eq. (1) since particle acceleration seems to be very weak or absent in Sgr A* (Markoff 2005). Recasting Eq. (1) as a Fokker-Planck equation, we used the fully implicit Chang-Cooper algorithm (Chang \& Cooper 1970; Chiaberge \& Ghisellini 1999) to solve it.

Once the time-evolved particle distribution solution had been obtained, we then computed the emitted SED due to angleaveraged synchrotron emission using Eq. (10) of Crusius \& Schlickeiser (1986) for relativistic electrons with Lorentz factor $\geq 2$, and using Eq. (8) of Katarzyński et al. (2006) for electrons in the cyclo-synchrotron regime with Lorentz factor $<2$. Following the prescription of Blumenthal \& Gould (1970), IC is computed by incorporating the Klein-Nishina correction for scattering beyond Thomson limit. The seed photons for IC are the photons produced locally by synchrotron emission (synchrotron self-Compton; SSC). In the absence of detailed spatial information about the hot stars constituting the central star cluster close to Sgr A* (see e.g., Genzel et al. 2000), we estimated emission from these stars by considering 100 stars each of temperature $10000 \mathrm{~K}$, situated 0.5 parsecs from Sgr A*. The photon density of locally produced synchrotron+SSC photons in the jet is at least 3 orders of magnitude higher than the total photon density from these stars, suggesting that external inverse Compton losses due to photons from the nearby cluster is not important.

Since the bulk motion of the emitting jet plasma is relativistic, we first compute the Doppler factor $\mathcal{D}=[\Gamma(1-\beta \cos \theta)]^{-1}$, where $\theta$ is the angle between the velocity and the line of sight, $c \beta$ is the bulk speed, and $\Gamma=1 / \sqrt{1-\beta^{2}}$ is the bulk Lorentz factor. The observed SED is then calculated by applying the appropriate special relativistic transformations to the emitted frequency and flux (see e.g., Lind \& Blandford 1985), and taking into account time-lags due to the spatial extent of the flow.

For any given frequency, when the flux from each jet segment is plotted as a function of distance from the black hole, it roughly resembles a bell-shaped curve (in the self-absorbed part of the jet). The full width at half maximum (FWHM) is an indicator of the size of the photosphere of the jet at this frequency. We used this procedure to calculate the frequency-size relationship.

In our model, the radio flares are caused by a perturbation in $\dot{M}$ at the jet-base, leading to a transient density enhancement. During a flare of duration $t_{\mathrm{d}}$ this density enhancement is quantified by the parameter $f_{\mathrm{n}}$ so that the number density at the base $\left(n_{0}\right)$ as a function of time is given by

$n_{0}(t)= \begin{cases}\left(1+f_{\mathrm{n}}\right) n_{0} & \left(0 \leq t \leq t_{\mathrm{d}}\right) \\ n_{0} & \text { otherwise. }\end{cases}$

As the overdensity propagates outward along the jet, the additional pressure in the overdense region should cool and expand into the neighboring segments of lower pressure and density. While a full computation of this diffusion (and resultant cooling) would require MHD simulations beyond the scope of 


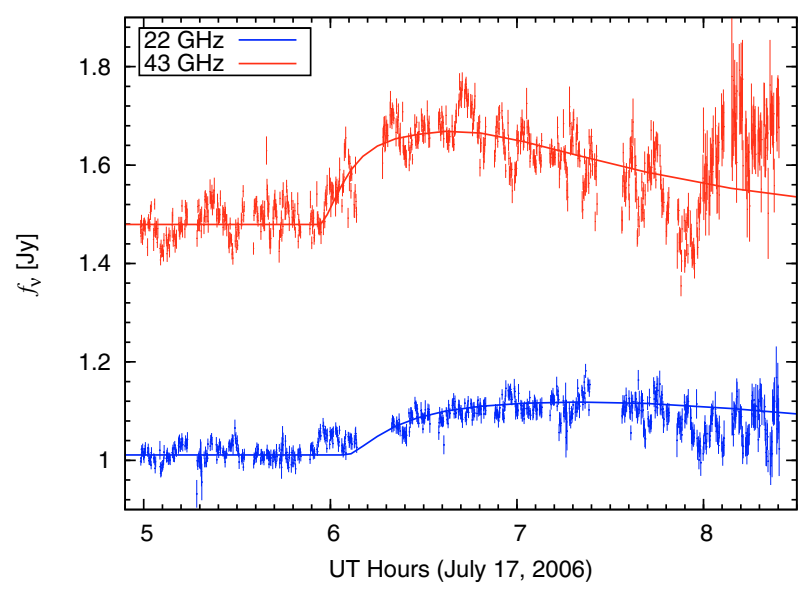

Fig. 1. Comparison of model with data for the flare on 2006 July 17 at 43 and $22 \mathrm{GHz}$. The $43 \mathrm{GHz}$ data with errorbars (from Yusef-Zadeh et al. 2008) are shown in red, and $22 \mathrm{GHz}$ data+model in blue. As noted in Sect. 3, we model the flare that created a peak in the $43 \mathrm{GHz}$ light curve close to $6.5 \mathrm{~h} \mathrm{UT}$.

the present paper, the effect can still be captured by assuming an additional velocity-dependent cooling term in the continuity equation for the overdense region. Assuming that the overdense region expands longitudinally into the neighboring segments with some fraction of the sound speed, we write this additional cooling term as a function of distance from the black hole $(z)$ as $\dot{\gamma} / \gamma=f_{\mathrm{o}} \beta_{\mathrm{s}} c / z$. Thus $f_{\mathrm{o}} \beta_{\mathrm{s}} c$ can be interpreted as the longitudinal speed with which the overdense region expands.

\section{Modeling the quiescent broadband emission and the radio flares from Sgr A*on July 17, 2006}

We compare the model predictions with the simultaneous observations of $\mathrm{Sgr} \mathrm{A}^{*}$ in 22 and $43 \mathrm{GHz}$ on 2006 July 17 (Yusef-Zadeh et al. 2008), which has the best simultaneous coverage to date of a flare at both frequencies. Indirect evidence suggests that $\mathrm{Sgr} \mathrm{A}^{*}$ is observed at a large inclination angle (Markoff et al. 2007; Falcke et al. 2009). For simplicity, we therefore assume that the jets are perpendicular to our line-ofsight, even though the model can accomodate any inclination angle. The free parameters of the model are: temperature $\left(T_{\mathrm{e}}\right)$ and density $\left(n_{0}\right)$ of the thermal leptons at the base, location of the sonic point or the nozzle $\left(h_{0}\right)$, radius of the nozzle $\left(r_{0}\right)$, and its magnetic field $\left(B_{0}\right)$. The flare is parametrized by its start time $\left(t_{0}\right)$, duration $\left(t_{\mathrm{d}}\right)$, density enhancement fraction $\left(f_{\mathrm{n}}\right)$, and expansion speed of the overdense region $\left(f_{\mathrm{o}}\right.$, which is a fraction of the sound speed).

The good agreement between the data and the model light curves is shown in Fig. 1. The model parameters are given in Table 1 . Note that we only model the flare that peaks around $6.5 \mathrm{~h}$ UT in $43 \mathrm{GHz}$. The increase in flux in both bands during the end of the observations may be the beginning of another flare as suggested by Yusef-Zadeh et al. (2008), but we do not model this due to lack of full coverage. In Fig. 2, we show modelpredicted light curves at $0.7,1.3,2,3.6,6$, and $13 \mathrm{~cm}$. From Fig. 1, it is clear that the light curves are asymmetric and even a phenomenological model would require assuming: (a) a rise timescale; (b) a decay timescale; and (c) an amplitude. In our model, three parameters also describe the flare completely, viz. $t_{\mathrm{d}}$ (which can be roughly associated with the rise time), $f_{\mathrm{o}}$ (associated with decay time), and $f_{\mathrm{n}}$ (associated with amplitude). It is thus important that a single set of flare parameters fits two rather
Table 1. Model parameters for the flare of 2006 July 17.

\begin{tabular}{llll}
\hline \hline Srl. No. & Parameter & Value & Unit \\
\hline 1 & $M_{\mathrm{BH}}^{*}$ & 4 & $10^{6} M_{\odot}$ \\
2 & Distance* $^{*}$ & 8.4 & $\mathrm{kpc}$ \\
3 & Inclination* $^{*}$ & 90 & degrees \\
4 & $r_{0}$ & 7 & $R_{\mathrm{g}}$ \\
5 & $h_{0}$ & 9 & $R_{\mathrm{g}}$ \\
6 & $B_{0}$ & 147 & $\mathrm{Gauss}$ \\
7 & $n_{0}$ & 7 & $10^{4} \mathrm{~cm}^{-3}$ \\
8 & $T_{\mathrm{e}}$ & 7 & $10^{10} \mathrm{~K}$ \\
9 & $t_{0}$ & 5.90 & $\mathrm{~h} \mathrm{UT}$ \\
10 & $t_{\mathrm{d}}$ & 1800 & $\mathrm{~s}$ \\
11 & $f_{\mathrm{n}}$ & 0.60 & \\
12 & $f_{\mathrm{o}}$ & 0.12 & \\
\hline
\end{tabular}

The mass $\left(M_{\mathrm{BH}}\right)$ and distance were taken from their latest estimates (see e.g., Gillessen et al. 2009; Reid et al. 2009). Inclination of the jet axis to the line of sight was fixed to 90 degrees. The base of the jet is parametrized by radius of the nozzle $\left(r_{0}\right)$, location of the sonic point $\left(h_{0}\right)$, magnetic field strength $\left(B_{0}\right)$, number density $\left(n_{0}\right)$, and temperature $\left(T_{\mathrm{e}}\right)$ of the thermal leptons. The flare is characterized by its start time $\left(t_{0}\right)$, duration $\left(t_{\mathrm{d}}\right)$, density enhancement fraction $\left(f_{\mathrm{n}}\right)$, and expansion speed of the overdense region $\left(f_{\mathrm{o}}\right.$, as a fraction of the sound speed). Parameters marked with an asterisk $\left(^{*}\right)$ were not varied during fitting.

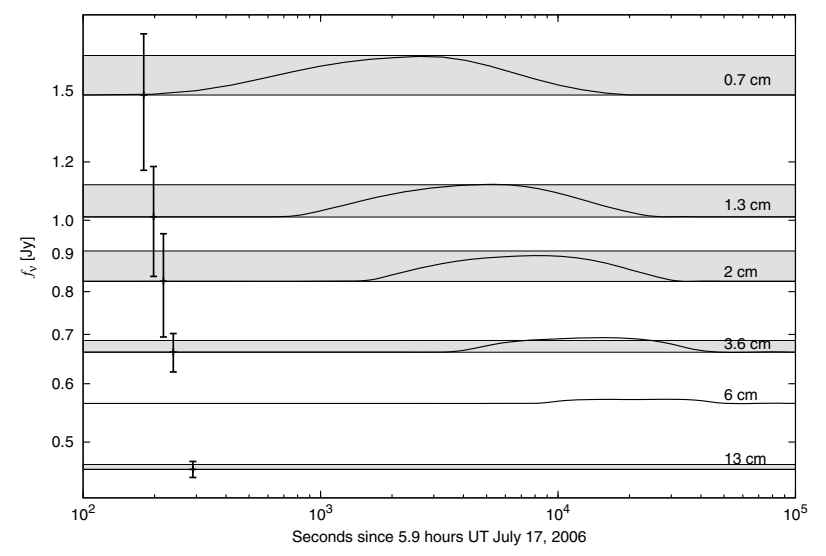

Fig. 2. Model-predicted light curves of Sgr A* at key radio frequencies using model parameters shown in Table 1. Also shown as errorbars between $t=200 \mathrm{~s}$ and $t=300 \mathrm{~s}$ are the rms flux variability of Sgr A* averaged over many flares as compiled by Falcke et al. (2009). The gray bands have a width of 0.6 times the rms variability of $\mathrm{Sgr} \mathrm{A}^{*}$, which is roughly matched to the specific flare under consideration.

disparate light curves at two frequencies. Moreover, the decrease in amplitude is consistent with the decrease in average variability with frequency. A compilation of the rms flux variability of Sgr A* averaged over many flares by Falcke et al. (2009) from VLA and GBI data (Falcke 1999a; Herrnstein et al. 2004) shows that the rms variability decreases with increasing wavelength. The relative amplitude of this flare at both 43 and $22 \mathrm{GHz}$ is about 0.6 times the long-term rms variability; therefore this flare was not extraordinarily bright or faint, and may be considered to be a fairly typical flare from Sgr A*.

The frequency-size relationship predicted by the model is displayed in Fig. 3, and shows reasonable agreement with the data at low frequencies where the jet becomes optically thick. The discrepancy at shorter wavelengths where the spectrum is optically thin is expected given that our model ignores general relativistic effects, and simplifies the physics of jet formation and launching, both of which must play an important role 


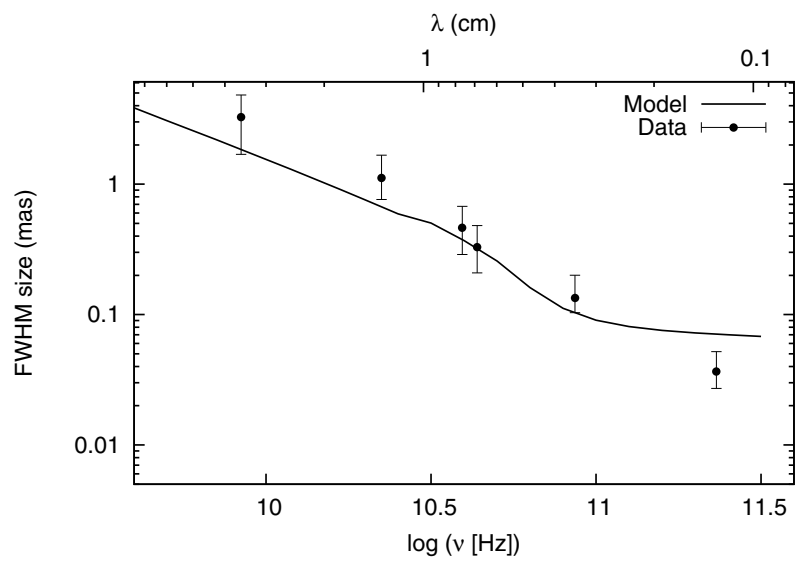

Fig. 3. Comparison of model-predicted frequency-size relationship (solid line) with observations. The data are from Bower et al. (2004), Shen et al. (2005), and Doeleman et al. (2008), which were compiled and corrected for scattering by Falcke et al. (2009).

very close to the black hole. For this model, the rate at which matter is channeled toward both jets is $\dot{M}=2 n_{0} m_{\mathrm{p}} c \beta_{\mathrm{s}} \gamma_{\mathrm{s}} \pi r_{0}^{2}=$ $2.4 \times 10^{-9} M_{\odot} \mathrm{yr}^{-1}$, which is about 2 orders of magnitude lower than the upper limit of $10^{-7} M_{\odot} \mathrm{yr}^{-1}$ suggested by linear polarization observations.

\section{Discussion}

We have shown that a time-dependent relativistic jet model can explain most of the observational features of $\operatorname{Sgr} \mathrm{A}^{*}$. The main conclusions of this work are:

- the model presented here can describe the quiescent broadband SED of Sgr A*, as well as its long-term radio variability;

- assuming that the radio flares are caused by a transient density enhancement at the base of the jet, which then propagates outward, the model can also fit the 22 and $43 \mathrm{GHz}$ light curve morphology of the flares seen on 2006 July 17 by Yusef-Zadeh et al. (2008). We predict light-curve morphology and delays at longer wavelengths, which can be used to test the model with future observations;

- the model predicted frequency-size relationship also matches the observed radio data quite well.

The radio emission in our model originates in the self-absorbed, optically thick part of the outflow, where adiabatic losses dominate over radiative cooling. Therefore, variability in the radio light curves largely traces the expansion of the jet plasma. Assuming that the flares have the same frequency-size relationship as the quiescent emission, the time-delay measurements combined with frequency-size measurements strongly constrain the bulk speed of the jet plasma in this model and exclude subrelativistic expansion speeds. An alternate model used by Yusef-Zadeh et al. (2008) to fit the radio light curves assumes adiabatic, subrelativistic expansion of a spherical blob of plasma, i.e. the classic van der Laan (1966) model. At the very least, our modeling shows that the van der Laan model is not unique, and that the jet model, in contrast to the van der Laan model, not only correctly predicts the light curves and the overall variability amplitudes, but also fits the frequency-size relation without violating any other observational constraints. It must be kept in mind that the jet model does not attempt to explain either the NIR or X-ray flares. The NIR/X-ray flares trace particle (re)energization and cooling very close to the black hole, while only marginally affecting the optically thick radio flux (Markoff et al. 2001), and no tight correlation between radio and X-ray flares have so far been found. A full general relativistic MHD model including radiative cooling (see e.g., Fragile \& Meier 2009; Mościbrodzka et al. 2009; Dexter et al. 2009) would be required to model the jet launching region. However, the simple model presented here captures the important physics and shows that the jet model explains the radio flaring properties naturally, because of the simple adiabatic expansion of overdensities in the outflow (likely linked to variations in the accretion rate). Future coordinated multiwavelength campaigns, especially measurements of time-lags, sizes and positional offset at other wavelengths, will enable a clearer understanding of the velocity profile of the jet from $\mathrm{Sgr}$ A*.

Acknowledgements. We would like to thank Farhad Yusef-Zadeh for providing us with the data of the radio light curves, and an anonymous referee for valuable comments.

\section{References}

Blandford, R. D., \& Königl, A. 1979, ApJ, 232, 34 Blumenthal, G. R., \& Gould, R. J. 1970, ApJ, 42, 237

Bower, G. C., Falcke, H., Herrnstein, R. M., et al. 2004, Science, 304, 704 Chang, J. S., \& Cooper, G. 1907, J. Comp. Phys., 6, 1

Chiaberge, M., \& Ghisellini, G. 1999, MNRAS, 306, 113

Crusius, A., \& Schlickeiser, R. 1986, A\&A, 164, L16

Dexter, J., Agol, E., \& Fragile, P. C. 2009, ApJ, 703, L142

Dodds-Eden, K., Porquet, D., Trap, G., et al. 2009, ApJ, 698, 676

Doeleman, S., Weintroub, J., Rogers, A. E. E., et al. 2008, Nature, 455, 78

Eckart, A., Schödel, R., García-Marín, M., et al. 2008, A\&A, 492, 337

Eckart, A., Baganoff, F. K., Morris, M. R., et al. 2009, A\&A, 500, 935 Falcke, H. 1996, ApJ, 464, L67

Falcke, H. 1999a, in The Central Parsecs of the Galaxy, ed. H. Falcke, A. Cotera, W. Duschl, F. Melia, \& M. J. Rieke, ASP Conf. Ser., 186, 113

Falcke, H. 1999b, in The Central Parsecs of the Galaxy, ed. H. Falcke, A. Cotera,

W. Duschl, F. Melia, \& M. J. Rieke, ASP Conf. Ser., 186, 148

Falcke, H., \& Biermann, P. L. 1999, A\&A, 342, 49

Falcke, H., \& Markoff, S. 2000, A\&A, 362, 113

Falcke, H., Markoff, S., \& Bower, G. C. 2009, A\&A, 496, 77

Fragile, P. C., \& Meier, D. L. 2009, ApJ, 693, 771

Gallo, E., Fender, R. P., Miller-Joner, J. C. A., et al. 2006, MNRAS, 370, 1351

Genzel, R., Pichon, C., Eckart, A., et al. 2000, MNRAS, 317, 348

Ghez, A. M., Salim, S., Weinberg, N. N., et al. 2008, ApJ, 689, 1044

Gillessen, S., Eisenhauer, F., Trippe, S., et al. 2009, ApJ, 692, 1075

Guilbert, P. W., Fabian, A. C., \& Rees, M. J. 1983, MNRAS, 205, 593

Herrnstein, R. M., Zhao, J.-H., Bower, G. C., et al. 2004, AJ, 127, 3399

Katarzyński, K., Ghisellini, G., Svensson, R., et al. 2006, A\&A, 451, 739

de Kool, M., Begelman, M. C., \& Sikora, M. 1989, ApJ, 337, 66

Leismann, T., Antón, L., Aloy, M. A., et al. 2005a, A\&A, 436, 503

Leismann, T., Antón, L., Aloy, M. A., et al. 2005b, A\&A, 436, 503

Lind, K. R., \& Blandford, R. D. 1985, ApJ, 295, 358

Liu, S., \& Melia, F. 2002, ApJ, 566, L77

Longair, M. S. 1992, High Energy Astrophysics (Cambridge University Press) Markoff, S. 2005, ApJ, 618, L103

Markoff, S., Falcke, H., Yuan, F., et al. 2001, A\&A, 379, L13

Markoff, S., Bower, G. C., \& Falcke, H. 2007, MNRAS, 379, 1519

Marrone, D. P., Moran, J. M., Zhao, J.-H., et al. 2007, ApJ, 654, L57

Marrone, D. P., Baganoff, F. K., Morris, M. R., et al. 2008, ApJ, 682, 373

Melia, F. 1992, ApJ, 387, L25

Mościbrodzka, M., Gammie, C. F., Dolence, J. C., Shiokawa, H., \& Leung, P. K. 2009, ApJ, in press [arXiv:0909.5431]

Nagar, N. M., Falcke, H., \& Wilson, A. S. 2005, A\&A, 435, 521

Narayan, R., Mahadevan, R., Grindlay, J. E., Popham, R. G., \& Gammie, C. 1998, ApJ, 492, 554

Reid, M. J., Menten, K. M., Zheng, X. W., et al. ApJ, 700, 137

Shen, Z.-Q., Lo, K. Y., Liang, M.-C., Ho, P. T. P., \& Zhao, J.-H. 2005, Nature, 438,62

van der Laan, H. 1966, Nature, 211, 1131

Yuan, F., Markoff, S., \& Falcke, H. 2002, A\&A, 383, 854

Yuan, F., Shen, Z.-Q., \& Huang, L. 2006, ApJ, 642, L45

Yusef-Zadeh, F., Wardle, M., Heinke, C., et al. 2008, ApJ, 682, 361

Yusef-Zadeh, F., Bushouse, H., Wardle, M., et al. 2009, ApJ, 706, 348 